\title{
Photonics for Radars Operating on Multiple Coherent Bands
}

\author{
(Invited Paper) \\ Paolo Ghelfi, Francesco Laghezza, Filippo Scotti, Daniel Onori, and Antonella Bogoni
}

\begin{abstract}
The introduction of photonics in microwave systems is setting new paradigms in radar architectures, providing new features potentially improving the surveillance effectiveness. In particular, photonics is enabling a new generation of multiband radars able to manage multiple coherent radar signals at different frequencies simultaneously, with high and frequencyindependent quality, enabling multispectral imaging for advanced surveillance systems. In fact, thanks to its high stability and huge bandwidth, photonics matches the urgent requirements of performance and flexibility of next generation softwaredefined radar architectures, and it guarantees system compactness thanks to the use of a single shared transceiver for multiband operations and to the potentials for photonic integration, which also promises reduced power consumption.

In this paper, we present the first field trial, in a maritime scenario, of a fully coherent multiband radar enabled by the use of photonics. The paper reviews the basic concepts exploited for the photonic generation and detection of the radar signals, and describes the extension to the multiband operation. We present details on the implementation and testing of a dual-band coherent radar system, discussing the potentials for a softwaredefined radio approach. Moreover, the results obtained after a simple digital data fusion are discussed, highlighting the capability of coherent photonics-based multiband radars in exploiting the extended observation bandwidth for improving the system detection resolution with minimum computational costs.
\end{abstract}

Index Terms-Doppler radar, microwave photonics, multiband radar, radar equipment, radar signal processing.

\section{INTRODUCTION}

$\mathrm{T}$ ODAY'S remote sensing systems are requiring increasingly demanding functionalities, putting electronic technologies under pressure. In fact, the applications of radar systems are broadening from mere ranging to new and multiple functions (recognition, tracking, mapping, etc.), also requiring multi-spectral sensing. These novel applications need higher performance in terms of sensitivity and resolution, entailing the necessity of very stable radiofrequency (RF) sources, and precise broadband signal detection and digitization. Multifunctional radar systems also ask for

Manuscript received on June XX, 2015.

P. Ghelfi, F. Laghezza, F. Scotti, and A. Bogoni are with CNIT - National Laboratory of Photonic Networks, Pisa, Italy (paolo.ghelfi@cnit.it).

D. Onori and A. Bogoni are with Scuola Superiore Sant'Anna - TECIP Institute, Pisa, Italy. reconfigurable and software-defined RF signal generators, capable of producing wideband waveforms over carriers ranging up to the millimeter waveband (MMW, above $30 \mathrm{GHz}$ ), while maintaining the phase stability necessary for coherent pulse-Doppler processing [1],[2]. Furthermore, multi-spectral system reconfigurability and reliability require a radar receiver with the digital back-end working up to the MMW range [3]. Wideband reconfigurable transmitters and receivers would therefore implement the software-defined radio (SDR) approach, allowing a single radar hardware to support different sensing signals. Nowadays, the digital generation of RF signals with acceptable stability is limited to few $\mathrm{GHz}$ by actual direct digital synthesizers (DDSs). Generating higher RF frequencies needs multiple upconversions, which worsen the signals phase noise [1],[2]. Today's analog-to-digital converters (ADCs) show a significant reduction of precision when the input bandwidth and sampling speed are increased [4]-[6], and signals at high carrier frequencies need to be down-converted before being digitized. So, the technical requirements for SDR radars are currently a serious challenge for high-speed DDSs and ADCs.

In the last decade, the research field of microwave photonics has been investigated as a possible solution to the several issues of electronic systems. Microwave systems can take advantage of the specific features of photonics, as wide bandwidth, immunity to electromagnetic interferences (EMI), low-loss and low-distortion propagation, low phase noise of optical clocks, and extremely high frequency flexibility. These unique properties translate in a wide range of advanced functionalities [7]-[10]. In particular, photonics allows generating RF signals in a wide range of carrier frequencies (up to the MMW) with superior phase stability. Photonics also enables the simultaneous generation of multiple RF carriers. It allows controlling the beam forming of wideband signals in phased array antennas (PAAs), with the capability for implementing a tunable filtering or other signal elaborations. At the receiver side, photonics permits the analog-to-digital conversion with large input bandwidth, high sampling rates, extremely low jitter, and the capability to receive multiple signals simultaneously. All these features candidate photonics as an enabler for SDR radar systems. Moreover, photonics allows the fiber-based distribution of the RF signals from the transceiver to the antenna site, with negligible transmission losses and distortions. The potential of using photonics in radar systems has been highlighted in recent works [11]-[14], evaluating the flexibility and high performance of a photonics- 
based transceiver, and testing photonics-based radars (incorporating single-frequency RF front-ends) in several aerial and maritime field trials.

The capability to work at different carrier frequencies is one of the fundamental requirements of SDR systems. This feature is necessary, for example, to trade off long distance detection and target tracking. In fact, while early-warning applications prefer S-band radars (strong immunity against weather clutter), target tracking is usually realized in the $\mathrm{X}$ band to generate narrower beams. Moreover, different materials show different reflectivity profiles along the RF range. The flexibility of adapting the radar carrier frequency would therefore allow optimizing the performance based on the operative conditions (weather, target distance, target material, required precision, etc.). A convenient way of implementing the frequency flexibility is the realization of a multiband system, and combining the information from multiple signal bands. In addition, multiband radars have a larger total bandwidth, and this can be used for improving the observation precision, merging the data from different bands [15]-[17]. Since photonics allows implementing coherent multiband systems with a single photonics-based transceiver, it holds the potential for simplifying the implementation of multiband radars, also reducing the system costs and improving the performance. We have recently developed the concept of photonics-based multiband radar in a preliminary study, realizing a dual-band transceiver that has been characterized in a laboratory environment [18]. In this paper, we present the results from the first field trial, in a maritime scenario, of the photonics-based dual-band radar demonstrator, and we analyze its performance and benefits. The demonstrator works simultaneously, coherently, and independently in the S- and X- bands, with a completely SDR approach. The design principles of the system architecture and the field trial results are detailed. The paper also demonstrates that the intrinsic coherence of the frequency bands enables a simplified data fusion, increasing the radar range resolution.

\section{PRINCIPLE OF OPERATION}

The scheme of the photonics-based multiband radar is reported in Fig. 1. The architecture is founded on a single photonics-based transceiver, composed by a multiband RF generator and a multiband RF receiver [18]. These functional blocks are both fed by a single mode locked laser (MLL). The MLL is a pulsed laser generating an optical spectrum composed of a series of modes, spaced by its pulse repetition rate $\Delta v=F_{M L L}$ and phase locked to each other, and it can be considered as a comb of coherent local oscillators. A digital waveform generator, providing simultaneous multiple signals at different intermediate frequencies (IFs), also feeds the RF generation block. As will be detailed in the next paragraphs, the RF generation block mixes the optical signal from the MLL with the electrical signals at IF, generating multiple RF signals at different frequency bands. Similarly, the RF receiver block combines the multiple received RF echoes with the optical signal from the MLL, obtaining the simultaneous down-conversion of each detected RF signal to its original IF. Then, an analog-to-digital converter (ADC) digitizes all the detected signals simultaneously, and a digital signal processor (DSP) elaborates the received data in the different bands. The scheme also includes a set of front-ends, one for each of the managed frequency bands, filtering the RF signals, boosting them before transmission, and pre-amplifying their received echoes. Although broadband front-ends are available, frequency selective RF front-ends show better performance and are therefore preferable for demanding applications.

In the proposed scheme, since the RF signals are all generated and detected using the same coherent laser comb, they are all intrinsically phase locked to each other. As will be detailed in the following, the proposed architecture therefore combines the capability for a multi-band SDR approach, with the potential for a simple fusion of the data from multiple bands and a consequent improvement of the system precision.

\section{A. Photonics-Based Multiband RF Generation}

For the sake of simplicity, here we consider the case of a dual-band radar. The generalization to the case of $n$ different bands is straightforward extending the following scheme. The conventional generation of a dual-band RF signal is performed using two different transmitters, each one based on multistage electronic up-conversions exploiting different RF local oscillators (LOs) (Fig. 2-top). Each up-conversion stage introduces phase and amplitude noise, due to the phase drifts of the LOs and to the use of noisy electronic mixers. The LOs are usually incoherent with each other. Moreover, their phase stability decreases as the RF gets higher.

On the contrary, the photonic RF generation of dual-band RF signals exploits a single transmitter (Fig. 2-bottom). The basic concept for the photonics-based RF generation is the heterodyning of two lasers in a photodiode, producing an RF signal whose frequency is equal to the detuning between the two beating lasers. This way, thanks to the wide optoelectrical bandwidth of the available commercial photodiodes, the generation of RF signals up to $100 \mathrm{GHz}$ is easily achievable. Moreover, if one of the beating lasers is modulated, the heterodyning operation up-converts the modulation to the detuning frequency. Since optical modulators are available with electro-optical bandwidth above $40 \mathrm{GHz}$, photonics allows the generation of ultra-broad bandwidth RF signals up to the MMW. In order to generate an RF signal with the phase stability required by surveillance applications, the beating lasers must be locked in phase with each other. To this extent, few techniques have been developed [19]-[21]. A particularly suitable solution for the generation of multiple signals is exploiting a comb of intrinsically phase-locked lasers, as a MLL [22]-[26]. Exploiting the stability of MLLs, we have recently proposed few techniques for generating multiple wideband RF signals with agile carrier frequencies selection [27],[28]. As reported in Fig. 2-bottom, to generate two RF signals simultaneously, three laser modes can be selected from the spectrum of the MLL. One mode is modulated by the sum of the two digitallygenerated independent signals at two different intermediate frequencies $f_{I F 1}$ and $f_{I F 2}$. The other two laser modes are selected with a detuning, respectively, of $N \Delta v$ and $M \Delta v$ from 
the first selected mode. This way, heterodyning all the three modes in a photodiode, several beatings are generated, and in particular the two desired RF signals $f_{R F I}=N \Delta v+f_{I F I}$ and $f_{R F 2}=M \Delta v+f_{I F 2}$, characterized by a strict phase coherence. Finally, two frequency-specific front-ends filter the two RF signals and boost them before irradiation from the antennas.

\section{B. Photonics-Based Multiband RF Receiver}

The conventional dual-band RF receiver (Fig. 3-top) exploits a structure analogous to the conventional transmitter. It performs multiple RF down-conversions on each detected signal to move them into the bandwidth of the ADC.

The photonics-based dual-band RF detection exploits the huge electro-optical bandwidth of a single optical modulator to move all the RF signals to the optical domain simultaneously. The technique is based on the photonic downconversion, as reported in Fig. 3-bottom. This basic scheme is dual to the photonic up-conversion reported in Fig. 2-bottom. To receive two RF signals simultaneously, three laser modes can be selected from the spectrum of the MLL. One mode is modulated by the sum of the two detected RF signals at frequencies $f_{R F 1}$ and $f_{R F 2}$. The other two laser modes are selected as close as possible to the two modulation sidebands of the first laser mode, i.e., with a detuning of $f_{I F 1}$ and $f_{I F 2}$ from the sidebands, respectively. Heterodyning the three modes in a photodiode, the two beatings at $f_{I F 1}$ and $f_{I F 2}$ are generated, and they can be detected simultaneously by an ADC [29].

\section{Photonics-BASEd Coherent DuAL-BAND RADAR IMPLEMENTATION}

We have implemented the photonics-based dual-band radar exploiting a MLL with a $F_{M L L}=400 \mathrm{MHz}$. A qualitative picture of the output optical spectrum of the MLL is shown in Fig. 4-A. At the transmitter side, a DDS generates the combination of two waveforms at different intermediate frequencies $f_{I F I}$ and $f_{I F 2}$ (Fig. 4-B). The implementation of the photonics-based transmitter is different from the operating principle reported in Fig. 2. In fact, the repetition rate of the MLL in the demonstrator would require customized filters to select single modes. Moreover, an integrated photonic structure would be required for stabilizing the scheme against polarization and phase variations induced by mechanical vibrations. Since the realization of such a custom integrated device is out of the scope of our analysis, we resort to a scheme that does not require separating the modes of the MLL, and can be implemented with off-the-shelf components [27]. In the implemented scheme, the whole spectrum of the MLL is amplitude modulated by the IF waveforms via a Mach-Zehnder modulator (MZM) (Fig. 4-C). This way, the IF signals are transferred as lower- and upper- sidebands around each optical mode of the MLL. When the optical signal thus modulated is detected by a photodiode, all the spectral components are heterodyned together. This way, a replica of the digitally-generated signals is obtained at any $k F_{M L L} \pm f_{I F n}$, with $k$ positive integer (Fig. 4-D), up to the bandwidth of the photodiode (in our set up, 12GHz). The output of the photodiode is then split onto two paths, where two electrical band-pass filters (BPFs) can extract the signals at the desired carrier frequencies (Fig. 4-E). Then, the two up-converted signals are boosted to be transmitted.

The received echo signals, which are opportunely filtered, amplified, and coupled together, feed the photonic receiver. As for the generation, the actual implementation of the photonics-based RF receiver differs from the basic scheme described in Fig. 3-bottom, in order to avoid the need of separating the modes of the MLL. In this case, the detected RF signals modulate the entire optical spectrum from the MLL by means of a MZM. This operation performs the direct and precise optical down-conversion of the RF signals (Fig. 4-F) [18]. Finally, a 400MSps 12-bit ADC digitizes the photodetected signal. If the transmitted waveforms have been both generated below the Nyquist frequency $F_{M L L} / 2$ (i.e., $<200 \mathrm{MHz}$ ), the under-sampling of the echoes at $400 \mathrm{MSps}$ does not produce overlapping of the different frequency bands.

A picture of the implemented photonics-based dual-band radar is reported in Fig. 5, where the main building blocks are highlighted. The two front-ends are also evident, in the X-and S- bands, with their related antennas. The X-band transceiver comprises a travelling-wave tube amplifier ensuring a peak power up to $100 \mathrm{~W}$, while the S-band front-end uses a solidstate amplifier of up to $10 \mathrm{~W}$ output power. The two parabolic antennas have a gain of $30 \mathrm{dBi}$ and a field of view of $10^{\circ}$.

It is important underlining that in the implemented scheme, the ADC and the DDS are synchronous. This detail avoids using reference signals to perform the Doppler analysis, since the received and digitized signals can be directly compared with the digital version of the transmitted signals at IF.

The photonics-based transceiver (without any frequencyselective front-end) has been characterized and compared with the performance of state-of-the-art electronic radar transceivers, as reported in [11]. The analysis has highlighted that the photonics-based transceiver already shows performance comparable to that of mature electronic systems in many important parameters. Moreover, photonics shows significant advantages in the extreme flexibility in the generated carrier frequency ranging up to $40 \mathrm{GHz}$ (while electronic radars have fixed frequency band), in the arbitrary modulation capability, and in the precision of the digitization (effective number of bits (ENOB) $>7$ up to $40 \mathrm{GHz}$, while electronic transceivers guarantee a direct digitization with comparable ENOB only up to few $\mathrm{GHz}$ ). These are fundamental requirements for the implementation of the SDR paradigm in surveillance systems, confirming photonics as a promising enabler for future radars.

It is worth noting that, if a photonic implementation based on the operating principles of Fig. 2 and Fig. 3 is adopted, a further improvement of the results can be expected. In fact, using only the necessary optical signals can translate into higher SNR of the desired RF or IF signals after the photodetection, and into lower distortions. Moreover, it also allows broadening the signal bandwidth. To this end, the realization of suitable ultra-narrow integrated optical filters is non-trivial, but few technical implementations have already been presented [30]. 


\section{EXPERIMENTAL SETUP AND RESULTS}

\section{A.Single-Band Performance Verification}

The photonics-based radar has been first characterized in the X-band only [11],[12]. In this implementation, the intermediate frequency has been set to $100 \mathrm{MHz}$, generating an RF signal at $9900 \mathrm{MHz}$, with RF filters bandwidth of $40 \mathrm{MHz}$. The field trial has been run in cooperation with GEM elettronica, in the area around the port of San Benedetto del Tronto, Italy. In this experiment, the detections obtained by the photonics-based radar system have been compared to the ones taken by a commercial coherent radar in the X-band, the SEAEAGLE by GEM elettronica [13],[31].

A laboratory comparison (see Table I) has highlighted very similar fundamental features for the two systems (including the front-ends and the DSPs): the minimum detectable signal (MDS) has turned out to be $-90 \mathrm{dBm}$ for the SEAEAGLE and $-87 \mathrm{dBm}$ for the photonics-based radar, while the noise figure has been measured to be respectively $5 \mathrm{~dB}$ and $8 \mathrm{~dB}$. These must be considered as promising results, being the photonicsbased radar at a demonstrator stage, realized with nondedicated photonic devices and off-the-shelf RF components.

The field trial comparison has been run acquiring the same maritime environment. The two radars have been set to transmit a linear chirp with a frequency deviation of $10 \mathrm{MHz}$ over a pulsewidth of $1 \mu \mathrm{sec}$ and a PRF of $10 \mathrm{kHz}$, and a transmitted peak power of $50 \mathrm{~W}$. The coherence integration time (CIT) has been set to $7.5 \mathrm{~ms}$, corresponding to the rotation of $1^{\circ}$ of the two rotating antennas exploited in these trials. The range resolution of the radar is easily $c / 2 B$, with $c$ the speed of light and $B$ the signal bandwidth. The linear chirp is typically chosen for allowing broad modulation bandwidths while minimizing the side lobes of the radar autocorrelation function. On the other hand, the speed resolution is equal to $1 / C I T$. The numbers above therefore ensure a resolution of $15 \mathrm{~m}$ in range and $2 \mathrm{~m} / \mathrm{s}$ in speed. Fig. 6-A shows the photonics-based radar detection trace overlaid to the satellite map of the port: the coastal area and the harbor shape are well represented, including the breakwater lines and a small boat at about 0.42 nautical miles (NM) with a detected radial velocity of 5 knots $(2.57 \mathrm{~m} / \mathrm{s})$. Fig. 6-B and $-\mathrm{C}$ report the plan position indicator (PPI) plots for the same scene taken by, respectively, the SEAEAGLE and the photonics-based radars. The plots show a perfect matching in the visualized scenario, demonstrating that the developed photonics-based radar is on par with state-of-the-art systems.

\section{B. Dual-Band Performance Verification}

The tests of the photonics-based radar in dual-band configuration have been recently run at the Center of Naval Support and Experimentation "Vallauri Institute" of the Italian Navy (CSSN-ITE), in Livorno (Italy). In this experiment, the intermediate frequencies generated by the DDS are at $f_{I F I}=59 \mathrm{MHz}$ and $f_{I F 2}=125 \mathrm{MHz}$, so that a signal in the S-band is realized at $f_{R F I}=2459 \mathrm{MHz}$ using the upper sideband of the $6^{\text {th }}$ laser harmonic, while the signal in the $\mathrm{X}$-band is at $f_{R F 2}=9875 \mathrm{MHz}$ selecting the lower sideband of the $25^{\text {th }}$ harmonic. The RF filters have a bandwidth of $20 \mathrm{MHz}$. The two radiating elements (in the $\mathrm{S}$ - and $\mathrm{X}$ - bands) are placed side by side, facing the sea area. An automatic identification system (AIS) receiver and a visual tracking station allow the pointing of the antennas and provide reference data of the nearby naval traffic. The two radar waveforms are both $5 \mu \mathrm{s}-$ long and $18 \mathrm{MHz}$-chirped pulses, with a PRI of $100 \mu \mathrm{s}$, and 200 pulses are collected in the $20 \mathrm{~ms}$ CIT. The S-band signal is transmitted with a time delay of $5 \mu \mathrm{s}$ (equal to the pulse duration) with respect to the X-band one, thus emulating a $10 \mu$ s-long radar pulse with a chirped bandwidth of $36 \mathrm{MHz}$.

Fig. 7 reports the range/velocity maps simultaneously detected by the S- and X-band radars while pointing at the same scene. Three targets are evident in both the acquisitions. Within these, one is a cooperating target provided by the CSSN-ITE (Target A), while the other two are noncooperating vessels cruising close to the port. In details, Target $\mathrm{A}$ is at about $1 \mathrm{NM}$ and moving away with a negative speed of about $5 \mathrm{kn}$; Target $\mathrm{B}$ is at anchor $(\mathrm{v}=0 \mathrm{kn})$ at $2.5 \mathrm{NM}$ from the radar; finally, Target $\mathrm{C}$ is at about $3 \mathrm{NM}$ and is approaching at about $10 \mathrm{kn}$. The data on position and speed have been compared with those provided by the AISs, confirming the correct detection of the two radar systems. It is also evident from Fig. 7 that, while the range resolution of the two graphs is equal (it depends on the bandwidth of the radar signal), the velocity resolution of the X-band radar is better than that in the S-band, since it depends on the signal carrier frequency. The speed resolution in the S-band is about $5.9 \mathrm{kn}$, while in the X-band it is reduced down to about $1.5 \mathrm{kn}$.

\section{DATA Fusion OF THE COHERENT DUAL-BAND RADAR}

The multispectral data from a multiband radar can also be used in a data fusion processing for improving the precision of the detection [15]-[17]. Complex algorithms allow merging the information from each band and synthesizing a new set of data corresponding to the union of the observation bands, thus ensuring a better resolution. The peculiar coherence of the signals in a photonics-based multiband radar allows simplifying this processing. In fact, the phase-coherent data from the different bands do not require complex phase alignment algorithms before being fused.

Fig. 8 reports the digital signal processing chain of the acquired data. The FPGA performs the complex digital down conversion (DDC) of the sampled data from the ADC, using the in-phase and quadrature components of two different numerical controlled oscillators (NCOs) at $f_{I F 1}$ and $f_{I F 2}$, respectively. The digital low pass filters (LPFs) separate the two channels, and the cascade integrated comb filters (CIC) decimate the flow of both complex data down to $25 \mathrm{MSps}$.

The logical flow of the radar processing, performed on the host PC, is the same for both the S- and X- channels (respectively, green block and blue block in Fig. 8). The data are organized in $\mathrm{MxN}$ matrices, with $\mathrm{M}$ the number of radar acquisitions and $\mathrm{N}$ the samples per acquisition. The data thus represented are filtered with the matched filter required by the transmitted waveform, and then they are FFT-processed by column to be coherently integrated across the $\mathrm{M}$ acquisitions. These operations allow the calculation of the range/velocity 
maps (RD: range/Doppler), as for example in Fig. 7, or any other post-processing (thresholding, imaging, tracking, etc.).

It is worth recalling that the SDR approach considers the possibility for the multiband system to adapt each signal according to the observed scene, changing the generated waveforms in terms of pulse shape, duration, bandwidth, and repetition rate [18]. These modifications therefore require the according change of the matrices dimensions and of the matched filters for each channel, that is easily implemented by reconfiguring the processing parameters via software.

Here we present a simple fusion algorithm that exploits the intrinsic coherence of the two data flows guaranteed by the photonics-based architecture. The method is based on the coarse approximation that two identical radar waveforms at different frequency bands are equally scattered by the observed targets, and are subject to equal losses during propagation. If this hypothesis can be accepted, the concatenation of two delayed chirped pulses with identical length $T_{i}$ and bandwidth $\Delta f$ produces a chirped pulse with length $2 T_{i}$ and bandwidth $2 \Delta f$, thus obtaining a doubled range resolution $\Delta R_{S+X}=c /(2 \cdot 2 \Delta f)$. The phase coherence of the $\mathrm{S}$ - and $\mathrm{X}$ - channels then (yellow block in Fig. 8) demands only for a Doppler shift correction (required by the different employed wavelength) and a time alignment. Finally, the synthetic signal is calculated by simply summing up the two echoes. For comparison, the data fusion for non-coherent signals requires iterative algorithms for aligning the phase of the involved signals, thus requiring heavy and long calculations [15]-[17].

The fusion technique described above has been applied to the collaborative target detected in the scenario of Fig. 7. Fig. 9-A shows a picture (side view) of the Target A, while Fig. 9$\mathrm{B}$ and Fig. 9-C report its range profile (highlighting only the main scatterers) detected singularly by the receivers in the Sand $\mathrm{X}$ - bands. It must be noted that Target $\mathrm{A}$ was moving away from the radar site during the detection, so the radar was seeing it from the stern. Thus, the target range profile approximately recreates the side view of the vessel. Both the $\mathrm{S}$ - and the $\mathrm{X}$ - bands detect a strong scatterer corresponding to the vessel's superstructure, while the stern is clearly visible only in the S-band profile, about $20 \mathrm{~m}$ behind (the total length of the vessel is $32 \mathrm{~m}$ ). Fig. 9-D shows the synthetic range profile calculated by fusing the data from the two bands, using the simplified algorithm described above. A much more detailed profile is obtained. Multiple scatterers can be seen on the stern (the winches), and the shape of the superstructure shows the mast separated from the backside bulkhead, according to the real form of the boat. As expected from our initial hypothesis, the range resolution of the synthetic data $(4 \mathrm{~m})$ is half that of the single bands $(8 \mathrm{~m})$, as it would be with a doubled observation bandwidth. Despite the coarse assumptions considered, the outcome of this very simple method appears promising, helping improving the detection and imaging precision with negligible processing costs, taking advantage of the availability of the two observation bands.

\section{CONCLUSION}

In this paper, we have reported the design and field trial characterization of a photonics-based multi-band radar. The demonstrator exploits a single photonic transceiver to manage two radar signals, in the S- and in the X-bands. The system has been tested in a real maritime field trial, detecting both cooperating and non-cooperating targets. The detection results highlight the potentials of multi-spectral observations, that can gather different information from different bands, and can combine the data to get an overall improved resolution and sensitivity through data fusion.

Moreover, we have pointed out that photonics-based multiband radars generate intrinsically coherent signals over the different bands, and this peculiar feature allows a very simplified data fusion processing. We have implemented this feature in our demonstrator, obtaining the doubling of the range resolution with negligible processing costs, limited to the simple summation of the data from the two signal bands.

Therefore, the advanced functionalities of photonics are making its use in future radars a viable solution for enabling the SDR systems, fulfilling the requests for smart adaptation and improved situational awareness.

\section{ACKNOWLEDGMENT}

The authors thank Dr. V. Malaspina and Dr. L. Banchi at GEM elettronica, and the CSSN-ITE, in particular Lieutenant Commanders T. Martini and R. Soleti, for their support in the measure campaigns.

\section{REFERENCES}

[1] M.L. Skolnik, "Introduction to radar systems", 2nd Ed., McGraw-Hill, New York, 1980.

[2] M. Richards, J. A. Scheer, W. A. Holm, "Principle of Modern Radar: basic principle", SciTech Publishing, 2010

[3] J.B. Tsui, "Digital Techniques for Wideband Receivers", 2nd Ed, SciTech Publishing, 2004.

[4] R. Walden, "Analog-to-digital conversion in the early twenty-first century", Wiley Encyclopedia of Computer Science and Engineering, 2008

[5] G.C. Valley, "Photonic analog-to-digital converters", Optics Express, vol. 15, no 5, pp. 1955-1982, 2007.

[6] P.W. Juodawlkis, J.C. Twichell, G.E. Betts, J.J. Hargreaves, R.D. Younger, J.L. Wasserman, F. J. O’Donnell, K.G. Ray, R.C. Williamson, "Optically sampled analog-to-digital converters", IEEE Trans. Microwave Theory Techn., vol. 49, no. 2, pp. 1840-1853, 2001.

[7] A.J. Seeds, C.H. Lee, M. Naganuma, "Guest editorial: Microwave photonics". IEEE J. Lightwave Technol., vol. 21, no. 12, 2003.

[8] C.H. Cox, E. I. Ackerman, "Microwave Photonics: past, present and future", International Topical meeting on Microwave Photonics, 2008.

[9] J. Capmany, D. Novak, "Microwave Photonics combines two worlds", Nature Photonics, vol. 1, no. 6, pp. 319-330. 2007.

[10] J. Yao, "Microwave photonics", IEEE J. Lightwave Technol., vol. 27, no. 22, pp. 314-335, 2009.

[11] P. Ghelfi, F. Laghezza, F. Scotti, G. Serafino, A. Capria, S. Pinna, D. Onori, C. Porzi, M. Scaffardi, A. Malacarne, V. Vercesi, E. Lazzeri, F. Berizzi, A. Bogoni, "A fully photonics-based coherent radar system", Nature, vol. 507, no. 7492, pp. 341-345, 2014.

[12] F. Scotti, F. Laghezza, G. Serafino, S. Pinna, D. Onori, P. Ghelfi, A. Bogoni, "In-field experiments of the first photonics-based softwaredefined coherent radar", J. Lightwave Technol., vol. 32, n. 20, 2014

[13] F. Laghezza, F. Scotti, G. Serafino, L. Banchi, V. Malaspina, P. Ghelfi, A. Bogoni, "Field evaluation of a photonics-based radar system in a maritime environment compared to a reference commercial sensor", IET Radar Sonar Navig., 10.1049/iet-rsn.2014.0513.

[14] P. Ghelfi, F. Laghezza, F. Scotti, G. Serafino, S. Pinna, D. Onori, C. Porzi, M. Scaffardi, A. Malacarne, V. Vercesi, E. Lazzeri, A. Bogoni, 
"Fully photonics-based radar demonstrator: concept and field trials", OFC 2015, M3E.6, Los Angeles, USA.

[15] M. Vespe, C. J. Baker, and H. D. Griffiths, "Automatic target recognition using multi-diversity radar," IET Radar Sonar Navig., vol.1, no.6, pp. 470-478, 2007.

[16] P. Van Dorp, R. Ebeling, and A.G. Huizing, "High resolution radar imaging using coherent multiband processing techniques," IEEE Radar Conference, Washington DC, 2010, pp. 981-986.

[17] X. Wei, Y. Zheng, Z. Cui, Q. Wang, "Multi-band SAR Images Fusion Using the EM Algorithm in Contourlet Domain," Fuzzy Systems and Knowledge Discovery, 2007. FSKD 2007.

[18] F. Scotti, F. Laghezza, P. Ghelfi, and A. Bogoni, "Multi-Band SoftwareDefined Coherent Radar Based on a Single Photonic Transceiver", IEEE Trans. Microwave Theory Techn., vol. 63, no. 2, pp. 546-552, 2015.

[19] J. Yu, Z. Jia, T. Wang, G.K. Chang, "Centralized Lightwave RadioOver-Fiber System With Photonic Frequency Quadrupling for HighFrequency Millimeter-Wave Generation", IEEE Photonics Technol. Lett., vol. 19, no. 19, pp. 1499-1501, 2007.

[20] S. Pan, J. Yao, "Wideband and frequency-tunable microwave generation using an optoelectronic oscillator incorporating a Fabry-Perot laser diode with external optical injection", Optics Lett., vol. 35, no. 11, 2010.

[21] L. Maleki, "The optoelectronic oscillator," Nature Photonics, vol. 5, no. 12, pp. 728-730, 2011.

[22] H. Chi, J.P. Yao, "An approach to photonic generation of high frequency phase-coded RF pulses", IEEE Photonics Technol. Lett., vol. 19, no. 10, pp. 768-770, 2007.

[23] I.S. Lin, J.D. McKinney, A.M. Weiner, "Photonic synthesis of broadband microwave arbitrary waveform applicable to ultrawideband communication", IEEE Microwave and Wireless Components Letters, vol. 15, no. 4, pp. 226-228, 2005.

[24] J. A. Nanzer, P.T. Callahan, M.L. Dennis, T.R. Clark Jr. "Photonic Signal Generation for Millimeter- Wave Communications", Johns Hopkins APL Technical Digest, vol. 30, no. 4, pp. 299-308, 2012.

[25] T. Yilmaz, C.M. DePriest, T. Turpin, J.H. Abeles, P.J. Delfyett, "Toward a Photonic Arbitrary Waveform Generator Using a Modelocked External Cavity Semiconductor Laser", IEEE Photonics Technol. Lett., vol. 14, no. 11, pp. 1608-1610, 2002.

[26] J. Chou, Y. Han, and B. Jalali, "Adaptive RF-Photonic Arbitrary Waveform Generator", International Topical Meeting on Microwave Photonics, pp. 1226-1229, 2002.

[27] P. Ghelfi, F. Scotti, F. Laghezza, A. Bogoni., "Phase Coding of RF Pulses in Photonics-Aided Frequency-Agile Coherent Radar Systems" IEEE J. Quantum Technol., vol 48, no. 9, pp. 1151-1157, 2012.

[28] P. Ghelfi, F. Scotti, F. Laghezza, A. Bogoni, "Photonic Generation of Phase-Modulated RF Signals for Pulse Compression Techniques in Coherent Radars", IEEE J. Lightwave Techn., vol. 30, no. 11, 2012.

[29] F. Laghezza, F. Scotti, P. Ghelfi, A. Bogoni, "Photonics-Assisted Multiband RF Transceiver for Wireless Communications", IEEE J. Lightwave Technol., vol. 32, no. 16, pp. 2896-2904, 2014.

[30] M.C. Tien, J.F. Bauters, M.J.R. Heck, D.T. Spencer, D.J. Blumenthal, and J.E. Bowers, "Ultra-high quality factor planar $\mathrm{Si}_{2} \underline{\underline{N}}_{4}$ ring resonators on Si substrates", Opt. Exp., vol. 19, no. 14, 2011.

[31] http://www.gemrad.com/products/military/radar.php 